\title{
The Extents of Neuroscience and Neuropsychology in the Study of Artificial Intelligence
}

\section{A Fredrick}

Clinical Psychologist, National Health Service (NHS), UK.

Type of Review: Peer Reviewed.

DOl: http://dx.doi.org/10.21013/jas.v13.n3.p1

How to cite this paper:

Fredrick, A. (2018). The Extents of Neuroscience and Neuropsychology in the Study of Artificial Intelligence. IRA International Journal of Applied Sciences (ISSN 2455-4499), 13(3), 35-38. doi:http://dx.doi.org/10.21013/jas.v13.n3.p1

(C) Institute of Research Advances.

\section{(cc) EY-No}

This work is licensed under a Creative Commons Attribution-Non Commercial 4.0 International License subject to a proper citation to the publication source of the work.

Disclaimer: The scholarly papers as reviewed and published by the Institute of Research Advances (IRA) are the views and opinions of their respective authors and are not the views or opinions of the IRA. The IRA disclaims of any harm or loss caused due to the published content to any party.

Institute of Research Advances is an institutional publisher member of Publishers International Linking Association Inc. (PILA-CrossRef), USA. The institute is an institutional signatory to the Budapest Open Access Initiative, Hungary advocating the open-access of scientific and scholarly knowledge. The Institute is a registered content provider under Open Access Initiative Protocol for Metadata Harvesting (OAI-PMH).

The journal is indexed \& included in CAS Source Index of Chemical Abstracts Service of American Chemical Society (USA), Index Copernicus (IC Value 85.27), WorldCat Discovery Service (USA), CrossRef Metadata Search (USA), WorldCat (USA), OCLC (USA), Open J-Gate (India), EZB (Germany) Scilit (Switzerland), Airiti (China), Bielefeld Academic Search Engine (BASE) of Bielefeld University, Germany, PKP Index of Simon Fraser University, Canada. 


\begin{abstract}
Artificial Intelligence (AI) is the buzz of modern-day computing. On a daily basis, we are witnessing newer developments in the name of AI. AI is the conceptual depiction of near-humanly responses to a given problem/task. The modern technology built upon the fifth-generation computing is adding more AI capabilities for IT-enabled technologies. The real question here is to identify if the AI can go a long way without its roots i.e. neuroscience or some other disciplines of study can also be complementary for the future development of the AI-enabled technologies. This paper is a concept review paper for the importance of neuroscience for the development of AI systems.
\end{abstract}

Keywords: Artificial Intelligence, Neuroscience, Neuropsychology, Computing

AI seeks to replicate the human brain and its responses (of the later) for the identified problems. The human brain is the study subject falling under the neuroscience where neuroscience is the discipline of studying the neurons embedded into the human brain and its respective mechanisms.

The fluctuations and movements of neurons in the human brain are the mirror of what a problem looks like and the past experience encoded into its mainframe enable them to decide on a solution outcome. That is all about how the human brain experiences something and takes a decision for those experienced circumstances.

\title{
Neuroscience as the basis for AI
}

What we call artificial intelligence is the concept of the artificial human brain. Just like, neuroscience, AI is the result of the experiences of past behaviours of humans. AI has neuron-like trigger points embedded into it which enable it to take the best possible decision for a given situation. But all this trigger mechanism is the results of humans' past experiences of what they faced and how they reacted in the best possible way for seeking an optimum solution.

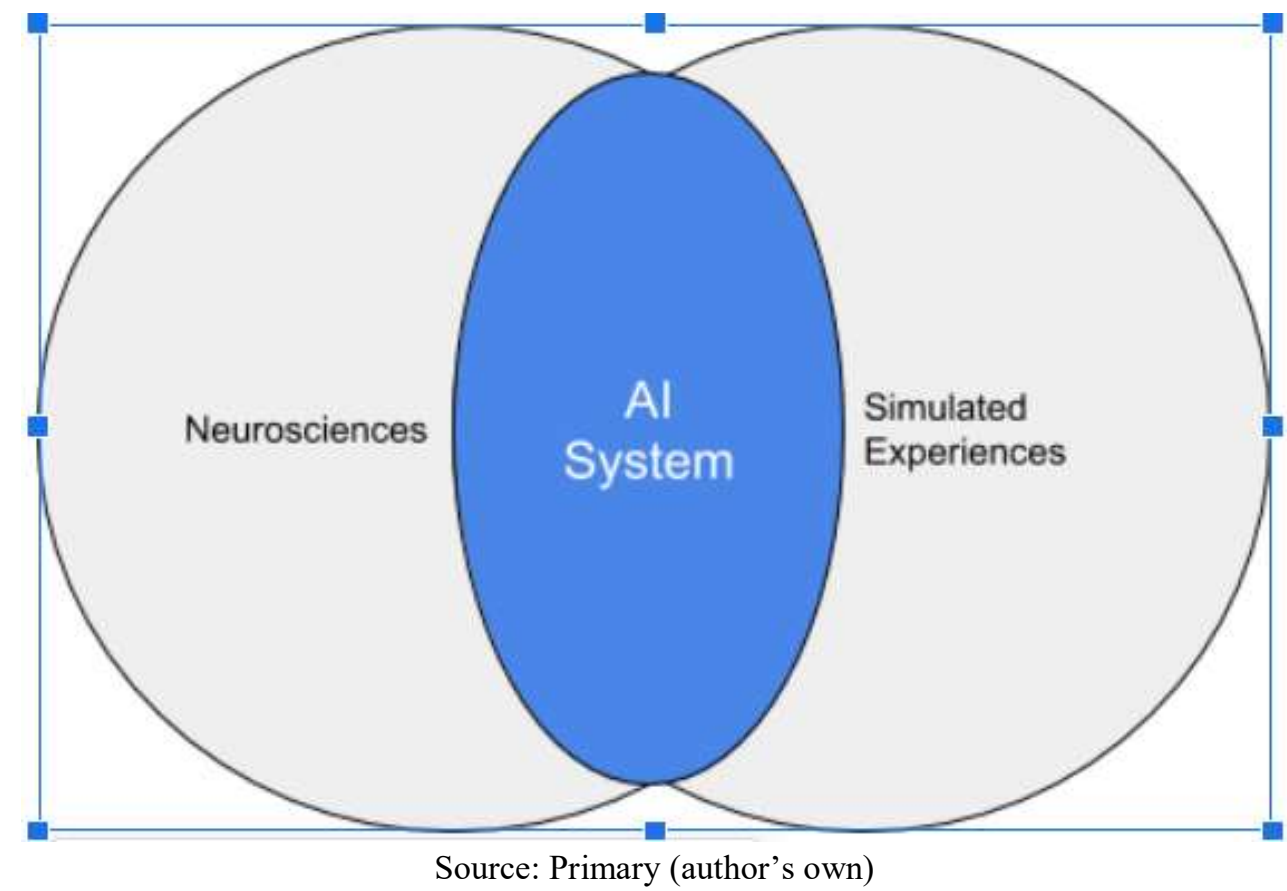

Figure 1: Depiction of the interpolation of neuroscience with simulated experiences leading to the creation of an AI system. 
The biggest limitation of AI is that it is based on past experiences. While the human brain is developing on an ongoing basis. All this necessitates the study of AI should be carried out in the light of developments in the neuroscience fronts.

\section{Neuropsychology as the enabler of neuroscience}

Neuropsychology, on the other hand, is the study of the interference of the neurons with human behavioural psychology. The neuropsychology has a lot to do with the neuroscience as the former derives the scientific justifications for the human brain's neuron reaction to any give psychological situation.

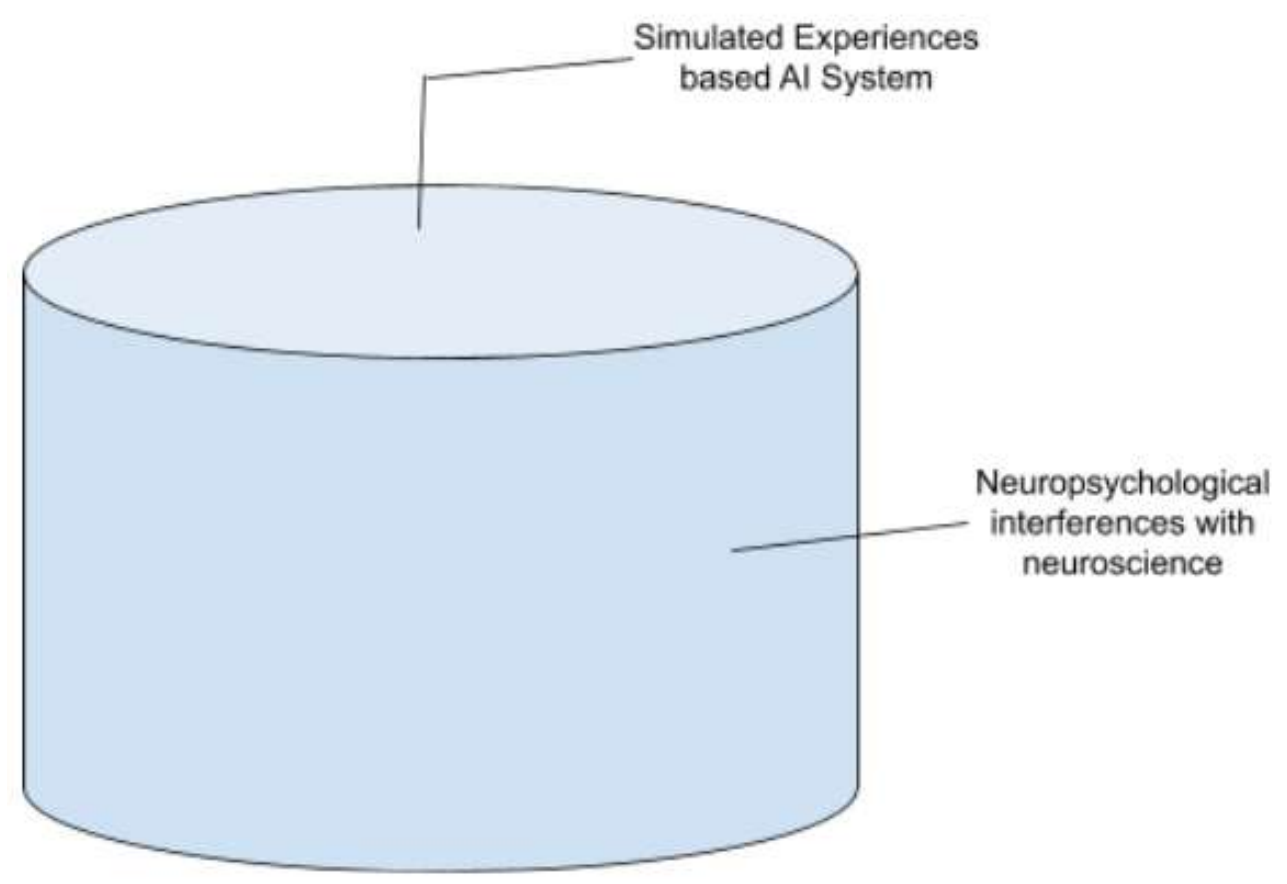

Source: Primary (author's own)

Figure 2: Depiction of synchronized efforts of neuropsychology and neuroscience for the creation of an AI System

Basically, the application of psychological replications in light of the scientific principles of neuroscience is the neuropsychology. Another important thing which makes the study of the neuropsychology even more important for the study of AI lies in the root linkages between the human brain's neuron and their responses to the real-life problems and solutions. The human behavioural psychology is built upon the given experiences as witnessed by the human brain over time. Collecting these experiences, neurons' reactions to these experiences and replicated psychological behavioural makes a perfect basis for building well trained artificial intelligence.

\section{Interference of neuropsychological advances on the AI}

As we have already seen that there are strong inter-linkages between neuropsychology and AI, which make it even more important to consider any achieved human behavioural psychological responses to a given situation where we want to apply AI. To make the AI successful for any given situation, we need to implement simulated human brain's responses to the problem and its possible solutions.

What is the need of the hour for the development of a reliable AI system is the legit and justified basis built upon neuropsychological experiences with the justifications of neuroscience. For achieving this, we need to take 
neuroscience, neuropsychology as well as the AI into one study group to conduct any fruitful simulation-based AI implementations.

\section{Future implications \& conclusion}

We shouldn't go studying the three separately. Instead, we need to regroup all these three disciplines into one study domain.

This way, a reliable, practical, well-refined and a continuous learning AI system can be built upon. Though the same will not replace the natural human neuron's capabilities but the same will at least be reliable in most of the identified responses scenarios.

\section{References}

[1]. Barbour, I. G. (1999). Neuroscience, artificial intelligence, and human nature: Theological and philosophical reflections. Zygon ${ }^{\circledR}, 34(3), 361-398$.

[2]. Chai, W. J., Abd Hamid, A. I., \& Abdullah, J. M. (2018). Working memory from the psychological and neurosciences perspectives: A review. Frontiers in psychology, 9, 401.

[3]. Hassabis, D., Kumaran, D., Summerfield, C., \& Botvinick, M. (2017). Neuroscience-inspired artificial intelligence. Neuron, 95(2), 245-258.

[4]. Reeke, G. N., \& Edelman, G. M. (1988). Real brains and artificial intelligence. Daedalus, 143-173. 